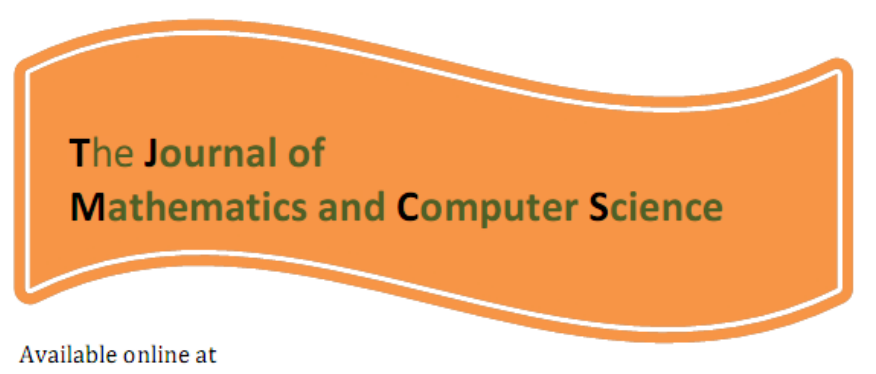

\title{
http://www.TJMCS.com
}

The Journal of Mathematics and Computer Science Vol. 4 No.1 (2012) 8 - 18

\section{Existence of positive solutions for third-order boundary value problems}

\author{
N. Nyamoradi * \\ Department of Mathematics, Faculty of Sciences \\ Razi University, 67149 Kermanshah, Iran.
}

Received: November 2011, Revised: March 2012

Online Publication: May 2012

\begin{abstract}
In this work, by employing the Guo-Krasnoselskii fixed point theorem, we study the existence of positive solutions to the third-order two-point non-homogeneous boundary value problem

$$
\left\{\begin{array}{l}
-u^{\prime \prime \prime}(t)=a(t) f(t, v(t)) \\
-v^{\prime \prime \prime}(t)=b(t) h(t, u(t)) \\
u(0)=u^{\prime}(0)=0, \alpha u^{\prime}(1)+\beta u^{\prime \prime}(1)=0 \\
v(0)=v^{\prime}(0)=0, \alpha v^{\prime}(1)+\beta v^{\prime \prime}(1)=0
\end{array}\right.
$$

where $\alpha \geq 0$ and $\beta \geq 0$ with $\alpha+\beta>0$ are constant.

Keywords: Positive solution, Two-point boundary value problem, Fixed point theorem.
\end{abstract}

\section{Introduction}

Third-order differential equations arise in a variety of different areas of applied mathematics and physics. Recently, the boundary value problems of third-order differential equations have received much attention. One may see Anderson [1], Anderson and Davis [2], Bai [3], Boucherif and Al-Malki [4], Graef and Yang [5], Grossinho and Minhos [6], Sun [13], Yao [14] and Yu et al. [15], and the references therein for related results. For example, in [1], Anderson obtained some existence results for positive solutions for the following BVP

$$
\begin{aligned}
x^{\prime \prime \prime}(t) & =f(t, x(t)) & t_{1} \leq t \leq t_{3} \\
x\left(t_{1}\right) & =x^{\prime}\left(t_{2}\right)=0, & \gamma x\left(t_{3}\right)+\delta x^{\prime \prime}\left(t_{3}\right)=0,
\end{aligned}
$$

by using the well-know Guo-Krasnoselshkii and Leggett-Williiams fixed point theorems [7, 10, 11]. In [13], Sun by the Guo-Krasnoselshkii fixed point theorem [7, 10] established various results on the existence of single and multiple positive solutions to some third-order differential equation satisfying the following three-point boundary conditions:

$$
x(0)=x^{\prime}(\eta)=x^{\prime \prime}(1)=0,
$$

\footnotetext{
*E-mail address: nyamoradi@razi.ac.ir. NN thanks Razi University for support.
} 
where $\eta \in\left[\frac{1}{2}, 1\right)$. In [8], Guo et al. obtained some existence results for at least one positive solution for the following BVP

$$
\begin{array}{rrr}
u^{\prime \prime \prime}(t) & +a(t) f(u(t))=0 & 0<t<1 \\
u(0) & =u^{\prime}(0)=0, & u^{\prime}(1)=\alpha u^{\prime \prime}(\eta),
\end{array}
$$

where $0<\eta<1$ and $1<\alpha<\frac{1}{\eta}$. In [9], Ling $\mathrm{Hu}$ et al. established result on the existence and multiplicity of positive solution for the following BVP:

$$
\left\{\begin{array}{l}
-u^{\prime \prime}(t)=f(x, v) \\
-v^{\prime \prime}(t)=g(x, u) \\
\alpha u(0)-\beta u^{\prime}(0)=0, \gamma u(1)+\sigma u^{\prime}(1)=0 \\
\alpha v(0)-\beta v^{\prime}(0)=0, \gamma v(1)+\sigma v^{\prime}(1)=0 .
\end{array}\right.
$$

In [12], Li Yunhong et al. considered the existence of positive solutions for the following BVP:

$$
\left\{\begin{array}{l}
-u^{\prime \prime \prime}(t)=a(t) f(t, v(t)) \\
-v^{\prime \prime \prime}(t)=b(t) h(t, u(t)) \\
u(0)=u^{\prime}(0)=0, u^{\prime}(1)=\alpha u^{\prime}(\eta) \\
v(0)=v^{\prime}(0)=0, v^{\prime}(1)=\alpha v^{\prime}(\eta)=0 .
\end{array}\right.
$$

Motivated greatly by the above-mentioned excellent works, in this paper we will consider the existence of positive solutions for the following nonlinear third- order two-point boundary value problem

$$
\left\{\begin{array}{l}
-u^{\prime \prime \prime}(t)=a(t) f(t, v(t)) \\
-v^{\prime \prime \prime}(t)=b(t) h(t, u(t)) \\
u(0)=u^{\prime}(0)=0, \alpha u^{\prime}(1)+\beta u^{\prime \prime}(1)=0 \\
v(0)=v^{\prime}(0)=0, \alpha v^{\prime}(1)+\beta v^{\prime \prime}(1)=0
\end{array}\right.
$$

where $\alpha \geq 0$ and $\beta \geq 0$ with $\alpha+\beta>0$ are constant.

Here, by a positive solution $u^{*}$ of BVP (5) we mean a solution $u^{*}$ of BVP (5) which satisfies $u^{*}>0$, $0<t<1$. We give the following assumptions:

$(\mathrm{H} 1) a, b:[0,1] \rightarrow[0, \infty)$ is continuous and

$$
\begin{aligned}
& 0<\int_{0}^{1} s\left(1-\frac{\alpha s}{\alpha+\beta}\right) a(s) d s<\infty, \\
& 0<\int_{0}^{1} s\left(1-\frac{\alpha s}{\alpha+\beta}\right) b(s) d s<\infty .
\end{aligned}
$$

(H2) $f, h:[0,1] \times[0, \infty) \rightarrow[0, \infty)$ is continuous.

Also, throughout this paper, $C[0,1]$ be the Banach space with norm $\|u\|=\max _{0 \leq t \leq 1}|u|$ and

$$
G(t, s)=\left\{\begin{array}{ll}
\frac{\alpha t^{2}(1-s)}{2(\alpha+\beta)}+\frac{\beta t^{2}}{2(\alpha+\beta)}, & t \leq s \\
\frac{\alpha t^{2}(1-s)}{2(\alpha+\beta)}+\frac{\beta t^{2}}{2(\alpha+\beta)}-\frac{(t-s)^{2}}{2}, & s \leq t
\end{array} .\right.
$$

Inspired and motivated by the works mentioned above, in this work we will consider the existence or nonexistence of positive solutions to BVP (5). We shall first give a new form of the solution, and then determine the properties of the Green's function for associated linear boundary value problems; finally, by employing the Guo-Krasnoselskii fixed point theorem, some sufficient conditions guaranteeing the existence of a positive solution. The rest of the article is organized as follows: in Section 2, we present some preliminaries and the Guo-Krasnoselskii fixed point theorem that will be used in Section 3. The main results and proofs will be given in Section 3. Finally, in Section 4, an example is given to demonstrate the application of our main result. 


\section{Preliminaries}

In this section, we present some notation and preliminary lemmas that will be used in the proofs of the main results.

Definition 1. Let $X$ be a real Banach space. A non-empty closed set $P \subset X$ is called a cone of $X$ if it satisfies the following conditions:

(1) $x \in P, \mu \geq 0$ implies $\mu x \in P$,

(2) $x \in P,-x \in P$ implies $x=0$.

Lemma 1. Let $u, v \in C^{+}[0,1]:=\{u \in C[0,1], u(t) \geq 0, t \in[0,1]\}$, then the unique solution of the $B V P(5)$ is given by

$$
\begin{aligned}
& u(t)=\int_{0}^{1} G(t, s) a(s) f(s, v(s)) d s \\
& v(t)=\int_{0}^{1} G(t, s) b(s) h(s, u(s)) d s
\end{aligned}
$$

where

$$
G(t, s)= \begin{cases}\frac{\alpha t^{2}(1-s)}{2(\alpha+\beta)}+\frac{\beta t^{2}}{2(\alpha+\beta)}, & t \leq s \\ \frac{\alpha t^{2}(1-s)}{2(\alpha+\beta)}+\frac{\beta t^{2}}{2(\alpha+\beta)}-\frac{(t-s)^{2}}{2}, & s \leq t\end{cases}
$$

Proof. In fact, if $u(t)$ is a solution of the BVP (5), then we may suppose that

$$
u(t)=-\frac{1}{2} \int_{0}^{t}(t-s)^{2} a(s) f(t, v(s)) d s+A t^{2}+B t+C .
$$

By the boundary conditions (5), we get $B=C=0$ and

$$
A=\frac{\alpha}{2(\alpha+\beta)} \int_{0}^{1}(1-s) a(s) f(s, v(s)) d s+\frac{\beta}{2(\alpha+\beta)} \int_{0}^{1} a(s) f(s, v(s)) d s .
$$

Therefore, BVP (5) has a unique solution

$$
\begin{aligned}
u(t)=-\frac{1}{2} \int_{0}^{t}(t-s)^{2} a(s) f(s, v(s)) d s & +\frac{\alpha t^{2}}{2(\alpha+\beta)} \int_{0}^{1}(1-s) a(s) f(s, v(s)) d s \\
& +\frac{\beta t^{2}}{2(\alpha+\beta)} \int_{0}^{1} a(s) f(s, v(s)) d s .
\end{aligned}
$$

Similarly, we also obtain (8). The proof is complete.

We need some properties of the function $G$ in order to discuss the existence of positive solutions. For convenience, we define

$$
g(s)=s\left(1-\frac{\alpha s}{\alpha+\beta}\right) \quad s \in[0,1]
$$

Lemma 2. For any $(t, s) \in[0,1] \times[0,1]$, we have

$$
0 \leq G(t, s) \leq g(s)
$$


Proof. First, we will show that $G(t, s) \geq 0$ for any $(t, s) \in[0,1] \times[0,1]$. Since it is obvious in case $0 \leq t \leq s \leq 1$, we only need to prove the case $0 \leq s \leq t \leq 1$. Now we suppose that $0 \leq s \leq t \leq 1$. Then

$$
\begin{aligned}
G(t, s) & =\frac{\alpha t^{2}(1-s)}{2(\alpha+\beta)}+\frac{\beta t^{2}}{2(\alpha+\beta)}-\frac{(t-s)^{2}}{2} \\
& =\frac{1}{2(\alpha+\beta)}\left[\alpha t^{2}(1-s)+\beta t^{2}-(\alpha+\beta)(t-s)^{2}\right] \\
& =\frac{-1}{2(\alpha+\beta)}\left[\alpha t^{2} s-\alpha t s+\alpha\left(s^{2}-t s\right)-\beta t s+\beta\left(s^{2}-t s\right)\right] \\
& =\frac{1}{2(\alpha+\beta)}[\alpha t s(1-t)+\alpha s(t-s)+\beta t s+\beta s(t-s)] \geq 0 .
\end{aligned}
$$

Next, we will prove that $G(t, s) \leq g(s)$ for any $(t, s) \in[0,1] \times[0,1]$.

In fact, for any fixed $s \in[0,1]$, it easy to see that

$$
G_{t}(t, s)=\left\{\begin{array}{ll}
\frac{\alpha t(1-s)}{(\alpha+\beta)}+\frac{\beta t}{(\alpha+\beta)}, & t \leq s \\
\frac{\alpha t(1-s)}{(\alpha+\beta)}+\frac{\beta t}{(\alpha+\beta)}-(t-s), & s \leq t
\end{array} .\right.
$$

If $t \leq s$, then

$$
G_{t}(t, s)=\frac{\alpha t(1-s)}{(\alpha+\beta)}+\frac{\beta t}{(\alpha+\beta)}=t\left(1-\frac{\alpha s}{\alpha+\beta}\right) \leq s\left(1-\frac{\alpha s}{\alpha+\beta}\right)=g(s) .
$$

If $s \leq t$, then

$$
\begin{aligned}
G_{t}(t, s) & =\frac{\alpha t(1-s)}{(\alpha+\beta)}+\frac{\beta t}{(\alpha+\beta)}-(t-s) \\
& =\frac{1}{\alpha+\beta}[\alpha s(1-t)+\beta s] \\
& =s\left(1-\frac{\alpha t}{\alpha+\beta}\right) \\
& \leq s\left(1-\frac{\alpha s}{\alpha+\beta}\right) \\
& =g(s) .
\end{aligned}
$$

Therefore,

$$
G_{t}(t, s) \leq g(s) \quad(t, s) \in[0,1] \times[0,1] .
$$

Then, for any $(t, s) \in[0,1] \times[0,1]$ we have

$$
G(t, s)=\int_{0}^{t} G_{\tau}(\tau, s) d \tau \leq \int_{0}^{t} g(s) d \tau=t g(s) \leq g(s) .
$$

So, we have the conclusion.

Lemma 3. For any $(t, s) \in[\tau, 1] \times[0,1]$, we have

$$
\gamma g(s) \leq G(t, s)
$$

where $\gamma=\frac{\beta}{2(\alpha+\beta)} \tau^{2}$, and $\tau$ satisfies $\int_{\tau}^{1} g(s) a(s) d s>0$. 
Proof. If $s=0$, then by Lemma 2, the result follows. Now suppose $(t, s) \in[\tau, 1] \times(0,1]$. Then for $\tau \leq t \leq s \leq 1$, by $(3)$, we have

$$
\frac{G(t, s)}{\frac{1}{2} g(s)}=\frac{\frac{\alpha t^{2}(1-s)}{2(\alpha+\beta)}+\frac{\beta t^{2}}{2(\alpha+\beta)}}{\frac{1}{2} s\left(1-\frac{\alpha s}{\alpha+\beta}\right)} \geq \frac{\frac{\alpha t^{2}(1-s)}{(\alpha+\beta)}+\frac{\beta t^{2}}{(\alpha+\beta)}}{\left(1-\frac{\alpha s}{\alpha+\beta}\right)} \geq \frac{\alpha t^{2}(1-s)}{(\alpha+\beta)}+\frac{\beta t^{2}}{(\alpha+\beta)} \geq \frac{\beta}{(\alpha+\beta)} t^{2} .
$$

On the other hand, for $0<s \leq t \leq 1$, by (10) we have

$$
G(t, s) \geq \frac{\beta t s}{2(\alpha+\beta)} \geq \frac{\beta t^{2} s}{2(\alpha+\beta)}
$$

Also by (6) and (11), we have

$$
\frac{G(t, s)}{\frac{1}{2} g(s)}=\frac{\frac{\beta t^{2} s}{2(\alpha+\beta)}}{\frac{1}{2} s\left(1-\frac{\alpha s}{\alpha+\beta}\right)} \geq \frac{\frac{\beta t^{2} s}{(\alpha+\beta)}}{\left(1-\frac{\alpha s}{\alpha+\beta}\right)} \geq \frac{\beta}{(\alpha+\beta)} t^{2} .
$$

Thus

$$
\frac{1}{2} \frac{\beta t^{2}}{(\alpha+\beta)} g(s) \leq G(t, s) \quad \forall(t, s) \in[\tau, 1] \times(0,1] .
$$

Therefore,

$$
\frac{1}{2} \frac{\beta \tau^{2}}{(\alpha+\beta)} g(s) \leq G(t, s) \quad \forall(t, s) \in[\tau, 1] \times(0,1] .
$$

Hence, we have the result.

Lemma 4. IF $u \in C^{+}[0,1]$, then the unique solution $u(t)$ of the $B V P(5)$ is nonnegative and satisfies $\min _{t \in[\tau, 1]} u(t) \geq \gamma\|u\|$.

Proof. It is obvious that $u(t)$ is nonnegative. For $t \in[0,1]$, by Lemma 2 , it follows that

$$
\begin{aligned}
u(t) & =\int_{0}^{1} G(t, s) a(s) f(s, v(s)) d s \\
& \leq \int_{0}^{1} g(s) a(s) f(s, v(s)) d s
\end{aligned}
$$

and therefore,

$$
\|u\| \leq \int_{0}^{1} g(s) a(s) f(s, v(s)) d s .
$$

On the other hand, for any $t \in[\tau, 1]$, from (7) and Lemma we obtain that

$$
\begin{aligned}
u(t) & =\int_{0}^{1} G(t, s) a(s) f(s, v(s)) d s \\
& \geq \gamma \int_{0}^{1} g(s) a(s) f(s, v(s)) d s .
\end{aligned}
$$

Hence,

$$
\min _{t \in[\tau, 1]} u(t) \geq \gamma\|u\| \text {. }
$$

Then, we achieve the desired result. 
Denote

$$
P=\left\{u \in C^{+}[0,1] ; \min u(t)_{t \in[\tau, 1]} \geq \gamma\|u\|\right\}
$$

It is obvious that $P$ is cone.

Define the operator $T_{1}$ and $T_{2}$ as follows

$$
\begin{aligned}
& T_{1} u(t)=\int_{0}^{1} G(t, s) a(s) f(s, v(s)) d s \\
& T_{2} v(t)=\int_{0}^{1} G(t, s) b(s) h(s, u(s)) d s
\end{aligned}
$$

for $t \in[0,1]$, it can be shown that $T_{1}: P \rightarrow X:=C[0,1]$ and $T_{2}: P \rightarrow X$ are continuous.

Lemma 5. The operator defined in (12) is completely continuous and satisfies $T_{1}(P) \subseteq P$.

Proof. The operator defined in (12) by an application of the Ascoli-Arzela theorem, is completely continuous and by Lemma 4 , we know that $T_{1}(P) \subseteq P$.

Our approach is based on the following Guo-Krasnoselskii fixed point theorem of cone expansioncompression type [10].

Theorem 1. Let $X$ be a Banach space and $P \subseteq X$ a cone in $E$. Assume $\Omega_{1}$ and $\Omega_{2}$ are open subsets of $X$ with $0 \in \Omega_{1}$ and $\bar{\Omega}_{1} \subset \Omega_{2}$. Let $T: P \bigcap\left(\bar{\Omega}_{2} \backslash \Omega_{1}\right) \rightarrow P$ be a completely continuous operator. In addition suppose either

(A) $\|T u\| \leq\|u\|, \forall u \in P \cap \partial \Omega_{1}$ and $\|T u\| \geq\|u\|, \forall u \in P \cap \partial \Omega_{2}$ or

(B) $\|T u\| \geq\|u\|, \forall u \in P \cap \partial \Omega_{1}$ and $\|T u\| \leq\|u\|, \forall u \in P \cap \partial \Omega_{2}$

holds. Then $T$ has a fixed point in $P \cap\left(\bar{\Omega}_{2} \backslash \Omega_{1}\right)$.

\section{$3 \quad$ Main results}

In this section, we discuss the existence of a positive solution of BVP (5). We give the following assumptions:

$$
\begin{aligned}
& \left(A_{1}\right) \quad \lim _{v \rightarrow o^{+}} \sup _{t \in[0,1]} \frac{f(t, v)}{v}=0, \quad \lim _{u \rightarrow o^{+}} \sup _{t \in[0,1]} \frac{h(t, u)}{u}=0, \\
& \left(A_{2}\right) \quad \lim _{v \rightarrow \infty} \inf _{t \in[0,1]} \frac{f(t, v)}{v}=\infty, \quad \lim _{u \rightarrow \infty} \inf _{t \in[0,1]} \frac{h(t, u)}{u}=\infty, \\
& \left(A_{3}\right) \quad \lim _{v \rightarrow o^{+}} \inf _{t \in[0,1]} \frac{f(t, v)}{v}=\infty, \quad \lim _{u \rightarrow o^{+}} \inf _{t \in[0,1]} \frac{h(t, u)}{u}=\infty, \\
& \left(A_{4}\right) \quad \lim _{v \rightarrow \infty} \sup _{t \in[0,1]} \frac{f(t, v)}{v}=0, \quad \lim _{u \rightarrow \infty} \sup _{t \in[0,1]} \frac{h(t, u)}{u}=0 .
\end{aligned}
$$

We will show BVP (5) has at least one positive solution when (A1) and (A2) or (A3) and (A4) are satisfied.

Theorem 2. Assume (A1) and (A2) or (A3) and (A4) are satisfied, then BVP (5) has at least one positive solution.

Proof. We divide the proof into two steps.

Step 1. Assume that (A1) and (A2) hold. Since (A1) holds, for $\epsilon>0$, there exists $1>R_{1}>0$ such 
that $f(t, v) \leq \epsilon v, h(t, u) \leq \epsilon u$, for each $(t, v) \in[0,1] \times\left[0, R_{1}\right]$ and $(t, u) \in[0,1] \times\left[0, R_{1}\right]$. Set $\Omega_{1}=\left\{u \in C[0,1]:\|u\|<R_{1}\right\}$ and let $\epsilon$ satisfies

$$
\max \left\{\int_{0}^{1} g(s) a(s) d s, \int_{0}^{1} g(s) b(s) d s\right\} \cdot \epsilon \leq 1 .
$$

Then, for any $u \in P \cap \partial \Omega_{1}$, from Lemma 2 and Lemma 5 and using (14) we have

$$
\begin{aligned}
T_{1} u(t) & =\int_{0}^{1} G(t, s) a(s) f(s, v(s)) d s \\
& \leq \int_{0}^{1} g(s) a(s) \epsilon v(s) d s \\
& \leq \epsilon \int_{0}^{1} g(s) a(s) \int_{0}^{1} G(s, r) b(r) h(r, u(r)) d r d s \\
& \leq \epsilon^{2}\|u\| \int_{0}^{1} g(s) a(s) d s \cdot \int_{0}^{1} g(r) b(r) d r \\
& \leq\|u\|,
\end{aligned}
$$

which implies that

$$
\left\|T_{1} u\right\| \leq\|u\| \quad \text { for } u \in P \cap \partial \Omega_{1} .
$$

On the other hand, since (A2) holds, for $\rho>0$, there exists $R_{2}>R_{1}$ such that $f(t, v) \geq \rho v, h(t, u) \geq$ $\rho u$, for $(t, v) \in[0,1] \times\left[\gamma R_{2}, \infty\right),(t, u) \in[0,1] \times\left[\gamma R_{2}, \infty\right)$. Set $\Omega_{2}=\left\{u \in C[0,1]:\|u\|<R_{2}\right\}$ and let $\rho$ satisfies

$$
(\rho \gamma)^{2} \gamma \int_{\tau}^{1} g(s) a(s) d s \cdot \int_{\tau}^{1} g(s) b(s) d s>1 .
$$

For any $u \in P \cap \partial \Omega_{2}$, by Lemma 4 one has $\min _{t \in[\tau, 1]} u(t) \geq \gamma\|u\|=\gamma R_{2}$. Thus, from (12) and (16) we can conclude that

$$
\begin{aligned}
T_{1} u(t) & =\int_{0}^{1} G(t, s) a(s) f(t, v(s)) d s \\
& \geq \rho \gamma \int_{\tau}^{1} g(s) a(s) v(s) d s \\
& \geq \rho \gamma \int_{\tau}^{1} g(s) a(s) \int_{\tau}^{1} G(s, r) b(r) h(r, u(r)) d r d s \\
& \geq(\rho \gamma)^{2} \int_{\tau}^{1} g(s) a(s) d s \cdot \int_{\tau}^{1} g(r) b(r) u(r) d r \\
& \geq(\rho \gamma)^{2} \gamma\|u\| \int_{\tau}^{1} g(s) a(s) d s \cdot \int_{\tau}^{1} g(r) b(r) d r \\
\geq & \|u\|,
\end{aligned}
$$

and thus

$$
\left\|T_{1} u\right\| \geq\|u\| \quad \text { for } u \in P \cap \partial \Omega_{2} .
$$

Therefore, by (15), (17) and the first part of Theorem 1 we know that the operator $T_{1}$ has a fixed point in $P \bigcap\left(\bar{\Omega}_{2} \backslash \Omega_{1}\right)$. Similarity, it can be proven that $T_{2}$ has a fixed point in $P \bigcap\left(\bar{\Omega}_{2} \backslash \Omega_{1}\right)$. 
Step 2. Assume that (A3) and (A4) hold. Since (A3) holds, for $A>0$, there exists $R_{3}>0$ such that $f(t, v) \geq A v, h(t, u) \geq A u$, for $(t, v) \in[0,1] \times\left[0, R_{3}\right],(t, u) \in[0,1] \times\left[0, R_{3}\right]$, where $A$ satisfies

$$
(A \gamma)^{2} \gamma \int_{\tau}^{1} g(s) a(s) d s \cdot \int_{\tau}^{1} g(s) b(s) d s>1 .
$$

So, for any $u \in P$ with $\|u\|=R_{3}$, we have

$$
\begin{aligned}
T_{1} u(t) & =\int_{0}^{1} G(t, s) a(s) f(s, v(s)) d s \\
& \geq A \gamma \int_{\tau}^{1} g(s) a(s) v(s) d s \\
& \geq A \gamma \int_{\tau}^{1} g(s) a(s) \int_{\tau}^{1} G(s, r) b(r) h(r, u(r)) d r d s \\
& \geq(A \gamma)^{2} \int_{\tau}^{1} g(s) a(s) d s \cdot \int_{\tau}^{1} g(r) b(r) u(r) d r \\
& \geq(A \gamma)^{2} \gamma\|u\| \int_{\tau}^{1} g(s) a(s) d s \cdot \int_{\tau}^{1} g(r) b(r) d r \\
& \geq\|u\|,
\end{aligned}
$$

and consequently, $\left\|T_{1} u\right\| \geq\|u\|$. So, if we set $\Omega_{3}=\left\{u \in P:\|u\|<R_{3}\right\}$, then

$$
\left\|T_{1} u\right\| \geq\|u\| \quad \text { for } u \in P \cap \partial \Omega_{3} .
$$

Now consider the assumption (A4) and consider four cases:

Case(i). Suppose $f, h$ are bounded, say $f(t, v) \leq M, h(t, u) \leq M$ for all $u, v \in[0, \infty)$. In this case we choose

$$
R_{4}=\max \left\{2 R_{3}, M \cdot \max \left\{\int_{0}^{1} g(s) a(s) d s, \int_{0}^{1} g(s) b(s) d s\right\}\right\},
$$

so that for any $u \in P$ with $\|u\|=R_{4}$, we have

$$
T_{1} u(t)=\int_{0}^{1} G(t, s) a(s) f(s, v(s)) d s \leq M \int_{0}^{1} g(s) a(s) d s \leq R_{4} .
$$

So, $\left\|T_{1} u\right\| \leq\|u\|$. Similarly, we also obtain $\left\|T_{2} v\right\| \leq\|v\|$ for any $v \in P$ with $\|v\|=R_{4}$.

Case(ii). Suppose $f$ is bounded and $h$ is unbounded, say $f(t, v) \leq M$ for all $v \in[0, \infty)$. Now, since $\lim _{u \rightarrow \infty} \sup _{t \in[0,1]} \frac{h(t, u)}{u}=0$, there exists $R_{0}>0$ such that

$$
h(t, u) \leq \mu u \quad \text { for } u \in\left[R_{0}, \infty\right),
$$

where $\mu>0$ satisfies

$$
\mu \cdot \int_{0}^{1} g(s) b(s) d s \leq 1
$$

If define

$$
q(r)=\max \{h(t, u): t \in[0,1], 0 \leq u \leq r\}
$$

we have that

$$
\lim _{r \rightarrow \infty} q(r)=\infty
$$


Let $R_{4}=\max \left\{2 R_{3}, R_{0}, M \cdot \int_{0}^{1} g(s) a(s) d s\right\}$ and be such that

$$
q(r) \leq q\left(R_{4}\right) \leq \mu R_{4} \quad \text { for } r \in\left[0, R_{4}\right]
$$

(We are able to do this since $q$ is unbounded.) For $u \in P$ with $\|u\|=R_{4}$, we have

$$
\begin{aligned}
T_{1} u(t) & =\int_{0}^{1} G(t, s) a(s) f(t, v(s)) d s \\
& \leq M \int_{0}^{1} g(s) a(s) d s \\
& \leq M \int_{0}^{1} g(s) a(s) d s \leq R_{4} .
\end{aligned}
$$

Thus, $\left\|T_{1} u\right\| \leq\|u\|$. for any $v \in P$ with $\|v\|=R_{4}$

$$
\begin{aligned}
T_{2} v(t) & =\int_{0}^{1} G(t, s) b(s) h(t, u(s)) d s \\
& \leq \int_{0}^{1} g(s) b(s) q\left(R_{4}\right) d s \\
& \leq \mu R_{4} \int_{0}^{1} g(s) b(s) d s \leq R_{4} .
\end{aligned}
$$

So, $\left\|T_{2} v\right\| \leq\|v\|$.

Case(iii). Suppose $f$ is unbounded and $h$ is bounded. This case similar to be case (ii).

Case(iv). Suppose $f$ and $h$ are unbounded, by assumption $\left(A_{4}\right)$, there exists $R_{0}>0$ such that

$$
f(t, v) \leq \mu v, h(t, u) \leq \mu u \quad \text { for } u, v \in\left[R_{0}, \infty\right),
$$

where $\mu>0$ satisfies

$$
\mu \cdot \max \left\{\int_{0}^{1} g(s) a(s) d s, \int_{0}^{1} g(s) b(s) d s\right\} \leq 1 .
$$

We can therefore choose

$$
R_{4}=\max \left\{2 R_{3}, R_{0}, M \cdot \max \left\{\int_{0}^{1} g(s) a(s) d s, \int_{0}^{1} g(s) b(s) d s\right\}\right\} .
$$

So, or any $u, v \in P$ and $\|u\|=R_{4},\|v\|=R_{4}, t \in[0,1]$, we can obtain $\left\|T_{1} u\right\| \leq\|u\|,\left\|T_{2} v\right\| \leq\|v\|$. Therefore, in either case we may put $\Omega_{4}=\left\{u \in P:\|u\|<R_{4}\right\}$; then

$$
\left\|T_{1} u\right\| \leq\|u\|,\left\|T_{2} v\right\| \leq\|v\| \quad \text { for } u, v \in P \cap \partial \Omega_{4} .
$$

Thus, by the second part of Theorem 1 we know that the operator $T_{1}$ has a fixed point in $P \bigcap\left(\bar{\Omega}_{4} \backslash \Omega_{3}\right)$. Similarity, it can be proven that $T_{2}$ has a fixed point in $P \bigcap\left(\bar{\Omega}_{4} \backslash \Omega_{3}\right)$. Therefore the BVP $(5)$ has at least one positive solution. Hence, we have the conclusion.

\section{Application}

Example 3. Consider the following boundary value problem system:

$$
\left\{\begin{array}{l}
-u^{\prime \prime \prime}(t)=\frac{1}{t \sqrt{1-t}}\left(v^{2}(t)\right), \\
-v^{\prime \prime \prime}(t)=\frac{1}{t \sqrt{1-t}}(u(t) \sqrt{u(t)}|\ln u(t)|), \\
u(0)=u^{\prime}(0)=0, u^{\prime}(1)+u^{\prime \prime}(1)=0, \\
v(0)=v^{\prime}(0)=0, v^{\prime}(1)+v^{\prime \prime}(1)=0,
\end{array}\right.
$$


where

$$
\begin{aligned}
a(t) & =b(t)=\frac{1}{t \sqrt{1-t}}, & \alpha=\beta=1, \\
f(t, v(t)) & =v^{2}(t), & h(t, u(t))=u(t) \sqrt{u(t)}|\ln u(t)| .
\end{aligned}
$$

It is not difficult to verify that

$$
\begin{aligned}
& 0<\int_{0}^{1} g(s) a(s) d s=\int_{0}^{1} g(s) b(s) d s=4<\infty, \\
& \lim _{v \rightarrow o^{+}} \sup _{t \in[0,1]} \frac{f(t, v)}{v}=0, \quad \lim _{u \rightarrow 0^{+}} \sup _{t \in[0,1]} \frac{h(t, u)}{u}=0, \\
& \lim _{v \rightarrow \infty} \inf _{t \in[0,1]} \frac{f(t, v)}{v}=\infty, \quad \lim _{u \rightarrow \infty} \inf _{t \in[0,1]} \frac{h(t, u)}{u}=\infty .
\end{aligned}
$$

Then by Theorem 2, system (20) has at least one positive solution.

\section{References}

[1] D. R. Anderson, Green's function for a third- order generalized right focal problem, Math. Anal. Appl. 288 (2003) 1-14.

[2] D. R. Anderson, J. M. Davis, Multiple solutions and eigenvalues for third-order right focal boundary value problems, J. Math. Anal. Appl. 267 (2002) 135-157.

[3] Z. Bai, X. Fei, Existence of triple positive solutions for a third order generalized right focal problem, Math. Inequal. Appl. 9 (2006) 437-444.

[4] A. Boucherif, N. Al-Malki, Nonlinear three-point third order boundary value problems, Appl. Math. Comput. 190 (2007) 1168-1177.

[5] J. R. Graef, Bo Yang, Multiple positive solutions to a three point third order boundary value problem, Discrete Contin. Dyn. Syst. 2005 (Suppl.) 1-8.

[6] M. R. Grossinho, F. M. Minhos, Existence result for some third order separated boundary value problems, Nonlinear. Anal. 47 (2001) 2407-2418.

[7] D. Guo, V. Lakshmikantham, Nonlinear problem in Abstract Cones, Academic Press, New York, 1988.

[8] L. J. Guo, J. P. Sun, Ya H. Zhao, Existence of positive solutions for nonlinear third-order three-point boundary value problems, Nonlinear Anal. 68 (2008) 3151-3158.

[9] L. Hu, L. L. Wang, Multiple positive solutions of boundary value problems for systems of nonlinear second-order differential equations, J. Math. Anal. Appl. 335 (2007) 1052-1060.

[10] M. A. Krasnoselskii, Positive solutions of operator equations, Noordhoff, Groningen, Netherlands, 1964.

[11] R. W. Leggett, L. R. Williams, Multiple positive fixed point of nonlinear operators on orderd Banach space, Indiana Univ. Math. J. 28 (1979) 673-688.

[12] Y. Li, Y. Guo, G. Li, Existence of positive solutions for systems of nonlinear third-order differential equations, Commun Nonlinear Sci Numer Simulat 14 (2009) 3792-3797. 
N. Nyamoradi / TJMCS Vol. 4 No. 1 (2012) 8 - 18

[13] Y. Sun, Positive solutions of singular third-order three-point boundary value problem, J. Math. Anal. Appl. 306 (2005) 589-603.

[14] Q. Yao, The existence and multiplicity of positive solutions of a third-order three-point boundary value problem, Acta Math. Appl. Sin. 19 (2003) 117-122.

[15] H. Yu, H. , Y. Liu, Multiple positive solutions to third-order three-point singular semipositone boundary value problem, Proc. Indian Sci. 114 (2004) 409-422. 